\title{
Neurofilament Transport Is Dependent on Actin and Myosin
}

\author{
Cheolwha Jung, Teresa M. Chylinski, Aurea Pimenta, Daniela Ortiz, and Thomas B. Shea \\ Center for Cellular Neurobiology and Neurodegeneration Research, Departments of Biological Sciences and Biochemistry, University of Massachusetts \\ Lowell, Lowell, Massachusetts 01854
}

\begin{abstract}
Real-time analyses have revealed that some newly synthesized neurofilament (NF) subunits translocate into and along axonal neurites by moving along the inner plasma membrane surface, suggesting that they may translocate against the submembrane actin cortex. We therefore examined whether or not NF axonal transport was dependent on actin and myosin. Perturbation of filamentous actin in $\mathrm{NB} 2 \mathrm{a} / \mathrm{d} 1$ cells with cytochalasin B inhibited translocation of subunits into axonal neurites and inhibited bidirectional translocation of NF subunits within neurites. Intravitreal injection of cytochalasin B inhibited NF axonal transport in optic axons in a dose-response manner. NF subunits were coprecipitated from NB2a/d1 cells by an anti-myosin antibody, and myosin colocalized with NFs in immunofluorescent analyses. The myosin light chain kinase inhibitor ML-7 and the myosin ATPase inhibitor 2,3-butanedione-2-monoxime perturbed NF translocation within NB2a/d1 axonal neurites. These findings suggest that some NF subunits may undergo axonal transport via myosinmediated interactions with the actin cortex.
\end{abstract}

Key words: actin; axonal transport (axoplasmic transport); cytoskeleton; motor; myosin; neurofilament

\section{Introduction}

To elaborate and maintain their axons, neurons must transport various components from their site of synthesis within perikarya into and along axons by a process referred to as "axonal transport." Axonal transport is divided into two broad categories termed "slow" and "fast" axonal transport (for review, see Hirokawa, 1993; Nixon, 1998; Brady, 2000; Gallant, 2000). The way in which neurons achieve these varying transport rates has been postulated to arise either from using motor complexes that translocate at different speeds (for review, see Hirokawa, 1998; Brady, 2000 ) or by varying the duration of association of cargo with a single transport system (Ochs, 1975).

Known motor proteins that mediate fast axonal transport include the kinesin and cytoplasmic dynein families. Kinesin translocates toward the "plus" end of microtubules (i.e., the end to which tubulin subunits predominantly are added), whereas cytoplasmic dynein translocates toward the "minus" end (from which subunits predominantly are removed) (Susalka and Pfister, 2000). Because microtubules within the axon are oriented with their plus ends facing away from the cell body (Heidemann et al., 1981; Baas et al., 1988), kinesin participates in anterograde transport (for review, see Vale and Fletterick, 1997; Hirokawa, 1998; Martin et al., 1999), whereas dynein participates in retrograde transport (Dillman et al., 1996a,b; Waterman-Storer and Salmon, 1997; Gross et al., 2000). To date, motor proteins responsible solely for slow axonal transport have not been identified.

\footnotetext{
Received May 3, 2004; revised July 16, 2004; accepted July 21, 2004

This research was funded by the National Science Foundation.

Correspondence should be addressed to Thomas B. Shea, Center for Cellular Neurobiology and Neurodegeneration Research, Departments of Biological Sciences and Biochemistry, University of Massachusetts Lowell, One University Avenue, Lowell, MA 01854. E-mail: Thomas_Shea@uml.edu.

D0I:10.1523/JNEUROSCI.1665-04.2004

Copyright $\odot 2004$ Society for Neuroscience $\quad$ 0270-6474/04/249486-11\$15.00/0
}

Anterograde axonal transport of neurofilaments (NFs) is regulated in part on the microtubule motor, kinesin (Yabe et al., 1999, 2000; Prahlad et al., 2000; Xia et al., 2003). Retrograde NF transport also is observed (Yabe et al., 1999; Roy et al., 2000; Wang et al., 2000), which is consistent with the interaction of NFs with a dynein-like motor (for review, see Brady, 2000; Shea and Flanagan, 2001). In this regard, dynein transports NFs along microtubules under cell-free conditions (Shah et al., 2000). The ability of fast axonal transport motors such as kinesin and dynein to translocate NF subunits despite their overall translocation at a rate consistent with slow transport (Nixon, 1992, 1998; Hirokawa, 1993; Galbraith and Gallant, 2000; Shea, 2000) is explained by the observation that NFs undergo short bursts of rapid transport that are interrupted by prolonged pauses (Roy et al., 2000; Wang et al., 2000; Ackerley et al., 2003).

The actin-based motor protein myosin also participates in axonal transport (Kuznetsov et al., 1992; Morris and Hollenbeck, 1995; Bridgman, 2004; Brown and Bridgman, 2004). Recent observations identify NFs as a major ligand of myosin in situ (Rao et al., 2002). NFs also have been shown to bind the actin-associated protein spectrin (Frappier et al., 1987, 1991). These observations, coupled with the observation that some NFs and punctate structures containing NF subunits translocate along the peripheral area of axonal neurites (Yabe et al., 2001a,b), leave open the possibility that the submembrane actin cortex and/or its associated motor myosin may facilitate axonal transport of NFs. We tested this hypothesis by perturbing the actin submembrane network and inhibiting myosin activity and by monitoring NF transport.

\section{Materials and Methods}

Cell culture and differentiation. Mouse NB2a/d1 neuroblastoma cells were cultured in DMEM (high glucose formulation) containing $10 \%$ fetal bovine serum, and axonal neurites were induced by the addition of $1 \mathrm{~mm}$ dibutyryl cAMP (dbcAMP) as described previously (Shea et al., 1988, 1990; Shea and Beermann, 1994) for 24 hr. For microscopic anal- 
ysis the cells were plated in $35 \mathrm{~mm}^{2}$ glass-bottom plates, and for immunoblot analyses the cultures were plated in $100 \mathrm{~mm}^{2}$ plates. For simplicity and clarity of writing only, dbcAMP-induced neurites are at some points referred to as axons, and the translocation of material into and along axonal neurites is referred to as axonal transport. It is recognized that these neuroblastoma cultures are only a model system and may lack critical features characteristic of neurons in culture or in situ.

Transfection and microinjection. Biotinylated NF-light (bNF-L) was prepared as described previously, mixed with $70 \mathrm{kDa}$ fluoresceinconjugated dextran tracer (Molecular Probes, Eugene, OR), and microinjected into cells that had been treated with dbcAMP for $3 \mathrm{~d}$ (Yabe et al., 1999). Additional cells were injected with tracer or buffer only. NF subunits microinjected under these conditions do not enter axons in appreciable levels because of injection pressure or diffusion alone but instead require active transport (Jung et al., 1998). Injected cells were located via fluorescein optics and then examined by phase-contrast microscopy. Cells exhibiting any obvious trauma resulting from microinjection were excluded from additional analyses.

Cells treated for $2 \mathrm{~d}$ with dbcAMP were transfected as described previously (Yabe et al., 1999) with $0.62 \mu \mathrm{g} / \mathrm{ml}$ of a construct expressing NF-M conjugated to enhanced green fluorescent protein (eGFP) at its C terminus (GFP-M) in $16 \mu \mathrm{g} / \mathrm{ml}$ Lipofectamine reagent (Invitrogen, San Diego, CA) for $4 \mathrm{hr}$ in $1 \mathrm{ml}$ of serum-free medium; after $2 \mathrm{~d}$ of dbcAMP treatment the cultures received an additional milliliter of serumsupplemented medium. In some cases we also used a construct encoding eGFP conjugated to the $\mathrm{N}$ terminal (generous gift from Dr. R. Liem, Columbia University, New York, NY), which, as described previously (Yabe et al., 2001a), yielded similar results; all images presented herein are derived from transfections with the use of C-terminally linked GFP.

Injection of radiolabel and harvesting of tissues. Murine retinal ganglion cells were radiolabeled in situ by the injection of $70 \mu \mathrm{Ci}$ of ${ }^{35} \mathrm{~S}$ methionine in a total volume of $0.2 \mu \mathrm{l}$ via a pulled glass capillary pipette into the vitreous of anesthetized mice (Shea et al., 1997). Mice were killed by cervical dislocation at 1 and $6 \mathrm{~d}$ after injection. Retinas were dissected away from the rest of the eye, and optic axons were dissected into $9 \times 1.1$ $\mathrm{mm}$ segments on a glass slide on dry ice. Retinas and segments from 5-11 mice were pooled and homogenized in $1 \%$ Triton X-100 in $50 \mathrm{~mm}$ Tris, pH 6.9, containing $2 \mathrm{~mm}$ EDTA, $1 \mathrm{~mm}$ PMSF, and $50 \mu \mathrm{g} / \mathrm{ml}$ leupeptin at $4^{\circ} \mathrm{C}$ by 50 strokes in a tight-fitting glass Teflon homogenizer (Shea et al., 1997). The Triton-insoluble cytoskeleton was sedimented by centrifugation at $15,000 \times g$ for $15 \mathrm{~min}$.

Manipulation of actin and myosin dynamics. To perturb filamentous actin in culture, we added $5 \mu \mathrm{M}$ cytochalasin B (cyto B) (Shea, 1990) to NB2a/d1 cells for the final $2 \mathrm{hr}$ of differentiation with dbcAMP. To perturb actin dynamics in situ, we coinjected cyto B (100 and $500 \mu \mathrm{M})$ intravitreally along with the radiolabel. No apparent toxicity was observed after the injection of these concentrations; mild toxicity was observed after coinjection of $5 \mathrm{~mm}$ cyto $\mathrm{B}$, and these mice were excluded from analyses. To perturb myosin light chain kinase activity, we added the kinase inhibitor ML-7 (previously used to assess the role of myosin in axonal transport) (Morris and Hollenbeck, 1995; Bridgman, 1999) and the myosin ATPase inhibitor 2,3-butanedione-2-monoxime (BDM) (Cramer and Mitchison, 1995) to cultures for $30 \mathrm{~min}$. Cells were treated with inhibitors immediately after microinjection and 18-24 hr after transfection (to allow for accumulation of sufficient GFP-M for reproducible analysis) (Yabe et al., 1999); in both cases, therefore, the cells were observed after a total of $3 \mathrm{~d}$ of dbcAMP treatment.

Immunofluorescence. To visualize bNF-L, we fixed the cultures at $1 \mathrm{hr}$ after microinjection with $4 \%$ paraformaldehyde in Tris-buffered saline (TBS), $\mathrm{pH} 7.4$, for $5 \mathrm{~min}$ at room temperature. Then the cultures were rinsed two times in TBS ( $5 \mathrm{~min} /$ rinse), rinsed three times ( $5 \mathrm{~min} /$ rinse) with $10 \mathrm{mg} / \mathrm{ml}$ sodium borohydride in TBS (to reduce autofluorescence), and then rinsed with TBS. Cultures were blocked for $30 \mathrm{~min}$ in TBS containing $1 \%$ bovine serum albumin (BSA) and $2 \%$ normal goat serum. Cultures then were incubated overnight at $4^{\circ} \mathrm{C}$ in TBS containing $1 \%$ BSA and a 1:100 dilution of rabbit anti-biotin. The next morning the cultures were rinsed three times with TBS, reblocked for $30 \mathrm{~min}$, rinsed one time with TBS, and then incubated for $30 \mathrm{~min}$ at $37^{\circ} \mathrm{C}$ in TBS containing 1\% BSA and a 1:150 dilution of Texas Red-conjugated goat anti- rabbit IgG. Cells were rinsed three times with TBS and stored at $4^{\circ} \mathrm{C}$ in TBS until they were visualized. Endogenous NF subunits were visualized with a polyclonal antibody (SMI-31) directed against phospho-epitopes common to NF-H and NF-M (Jung et al., 1998). Myosin was visualized with a rabbit polyclonal anti-myosin antibody directed against total myosin (Sigma, St. Louis, MO).

Filamentous actin was visualized by staining with rhodaminephalloidin (Molecular Probes). Cultures were rinsed two times with PBS and fixed as described above. Samples were rinsed again with PBS, extracted for $5 \mathrm{~min}$ with $-20^{\circ} \mathrm{C}$ acetone, and air-dried. Rhodaminephalloidin was dissolved in methanol, evaporated to dryness, and then redissolved in $400 \mu \mathrm{l}$ of PBS according to the manufacturer's instruc-
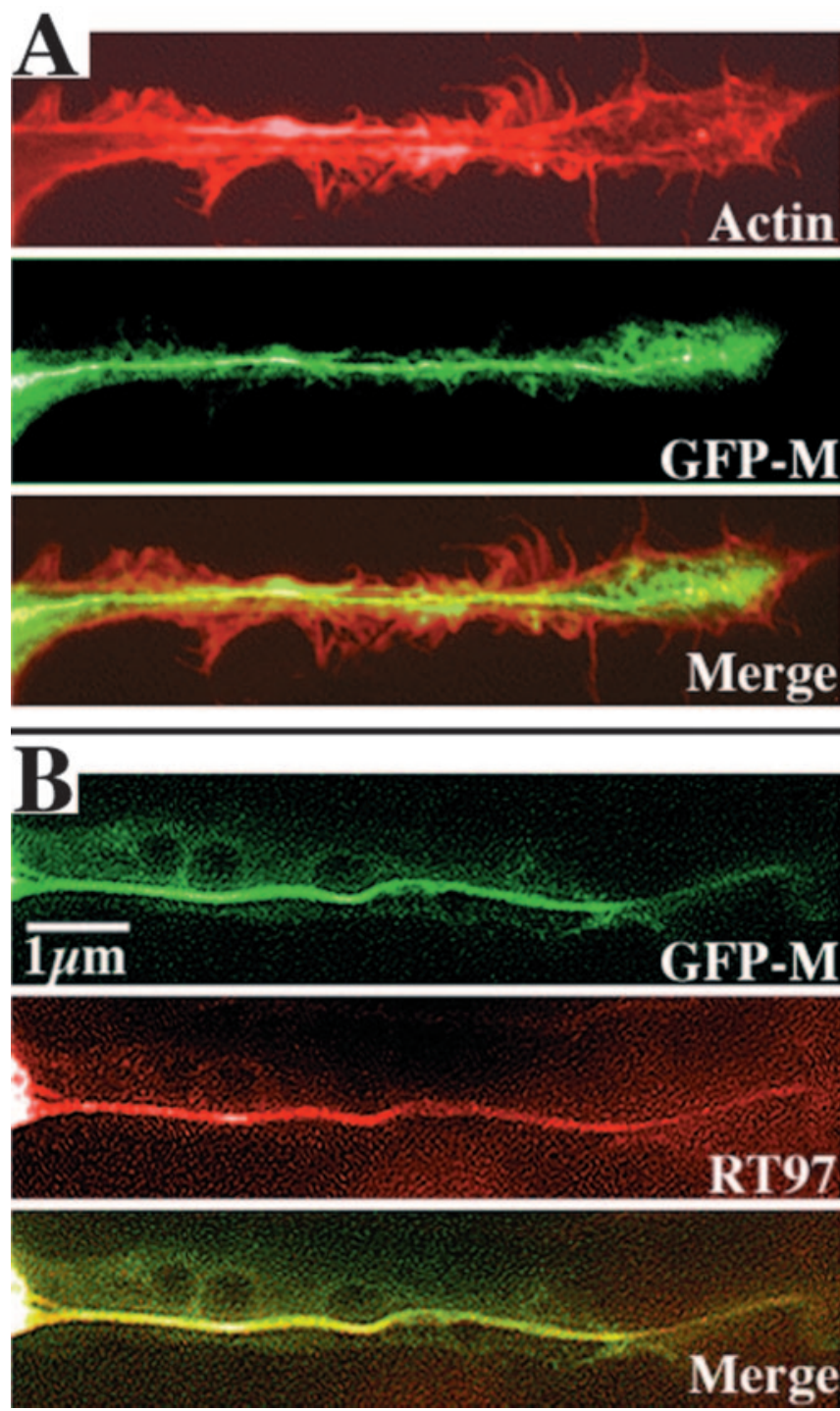

Figure 1. Axonal cytoskeletons display an array of filamentous actin and NFs and incorporate GFP-tagged NF subunits into the endogenous NF network. The panels present axonal neurites of two cells transiently transfected $24 \mathrm{hr}$ previously and then fixed and extracted with Triton X-100. The top cell $(A)$ was reacted with rhodamine-conjugated phalloidin to visualize filamentous actin, and the bottom cell ( $B$ ) was reacted with a monoclonal antibody (RT97) directed against a phosphorylated NF epitope. Note the peripheral concentration of filamentous actin. Note that both actin and GFP fluorescence are present along the entire length of the axonal neurite; however, actin is concentrated along the periphery, whereas GFP-M is concentrated along the central aspect of the neurite. This central concentration corresponds to the bundle of phospho-NFs (Yabe et al., 2001a,b) as confirmed in the bottom neurite by preferential labeling of this bundle by RT97. Also note the colocalization of GFP-tagged NF subunits with phospho-NFs in the merged image, indicating their incorporation into the NF cytoskeletal network. 

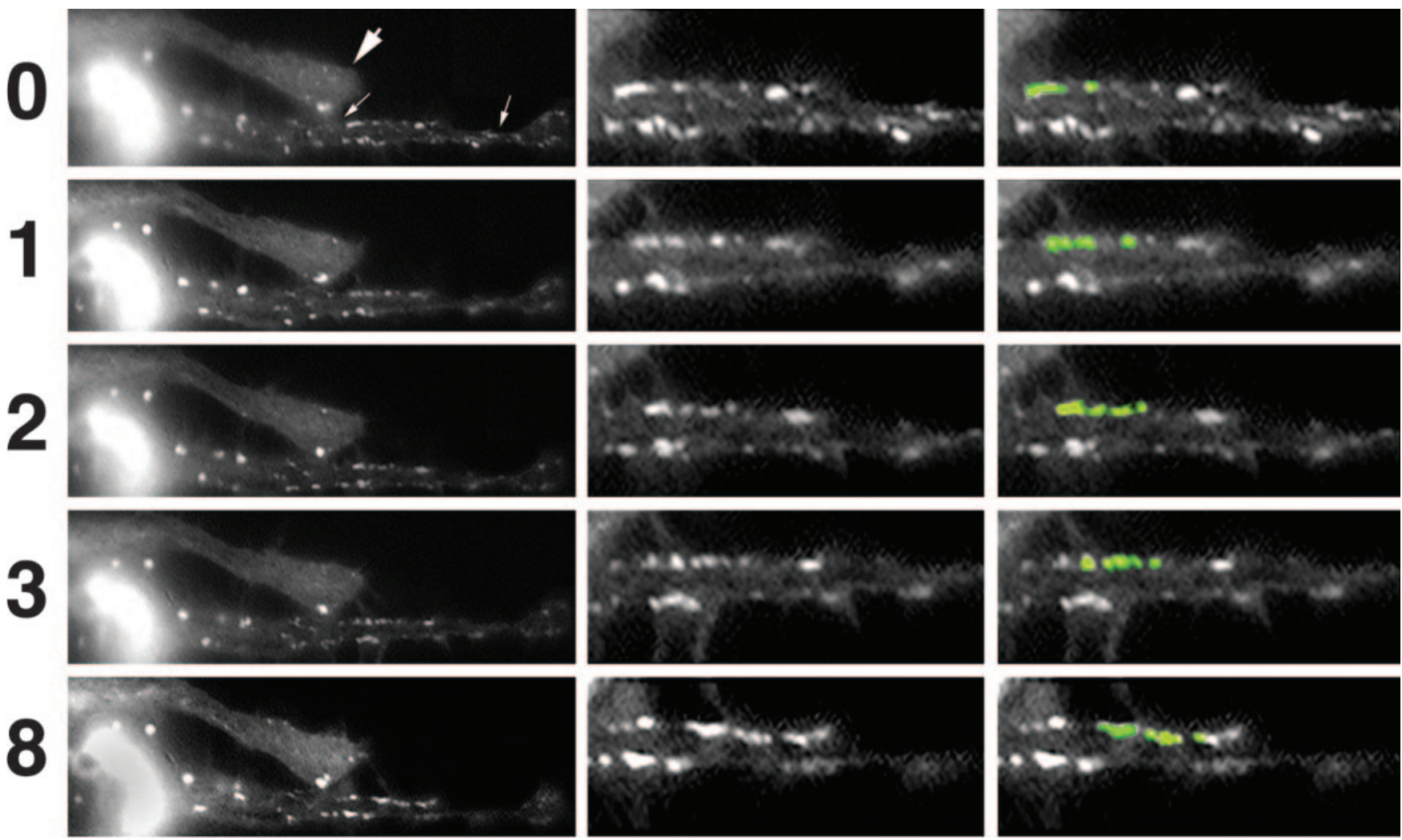

Figure 2. GFP-tagged NFs and punctate structures undergo bidirectional translocation along the inner plasma membrane of axonal neurites. Images captured sequentially under fluorescein optics at the indicated intervals (in minutes) are presented. The left panels present the axonal neurites of cells transfected with GFP-M. Small arrows denote the region of a cell in which GFP-tagged subunits have not yet incorporated throughout the entire endogenous NF network, which facilitates observation of particles undergoing translocation along the membrane; the region denoted by arrows is presented at higher magnification in the middle panels and after false coloration of select particles in the right panels. The large arrow to the left denotes the neurite of a second transfected cell in which GFP-tagged subunits previously have incorporated throughout the axonal NF network; note that this obscures the localization and movement of individual GFP-tagged structures during the analysis of sequential images. False color to highlight the video sequence corresponding to these images is presented in QuickTime format (supplemental material, available at www.jneurosci.org).
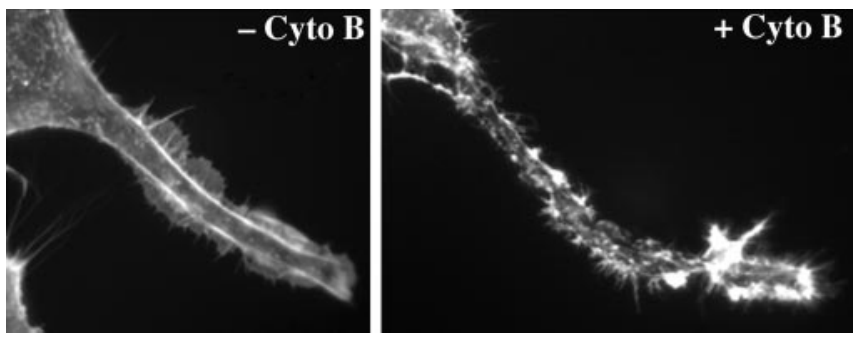

Figure 3. Cytochalsin B treatment disrupts the submembrane actin network. The panels present portions of perikarya and axonal neurites of cells before (left) and after (right) $2 \mathrm{hr}$ of treatment with $5 \mu \mathrm{m}$ cytochalasin B. Note the continuous distribution of actin-rich filamentous profiles in untreated cells and their perturbation after cytochalasin B treatment.

tions. Cultures were incubated with this solution for $20 \mathrm{~min}$ at room temperature, rinsed two times with $\mathrm{PBS}$, and stored in $\mathrm{PBS}$ at $4^{\circ} \mathrm{C}$ in the dark until visualization.

Immunoprecipitation. Cells were rinsed two times with cold TBS and scraped from the plate on ice in $50 \mathrm{~mm}$ Tris, $\mathrm{pH}$ 7.4, containing $5 \mathrm{~mm}$ EDTA, $2 \mathrm{~mm}$ PMSF, $50 \mu \mathrm{g} / \mathrm{ml}$ leupeptin, and $1 \%$ Triton X-100. Homogenates were incubated overnight at $40^{\circ} \mathrm{C}$ by shaking with a polyclonal antibody (R39) that reacts with all three NF subunits regardless of phosphorylation state (Shea et al., 1997) or with anti-myosin, followed by incubation for $2 \mathrm{hr}$ with $10 \mathrm{mg}$ of protein A-Sepharose (Sigma) as described previously (Shea et al., 1990, 1997); in some experiments SDS was omitted during incubation with anti-myosin to allow for potential coprecipitation of NF subunits along with myosin (Yabe et al., 2000). Immunoprecipitated material then was subjected to SDS-gel electrophore- sis and immunoblot analyses as described below. Additional samples were processed for immunoprecipitation in the absence of primary antibody (i.e., with protein A-Sepharose alone), which did not precipitate myosin or NF subunits.

Gel electrophoresis and immunoblot analyses. Samples received an equal volume of $2 \times$ Laemmli treatment buffer, were boiled for $1 \mathrm{~min}$, and were electrophoresed on linear 7\% polyacrylamide SDS gels; either the samples were dried and placed against Kodak (Rochester, NY) X-OMAT film to generate autoradiographs (Jung et al., 1998), or the separated proteins were transferred to nitrocellulose. Nitrocellulose replicas were probed as described previously (Shea et al., 1997) with 1:1000 dilutions of anti-myosin or SMI-31, followed by goat anti-rabbit or anti-mouse IgG conjugated to alkaline phosphatase as described previously (Jung et al., 1998).

Image acquisition and densitometric analysis. Epifluorescent and corresponding phase-contrast images were captured with a DAGE CCL-72 camera and stored in a Macintosh PowerPC 7100AV via NIH Image software and a Scion (Frederick, MD) LG-3 frame grabber. Images were stored as TIFF or PICT files and analyzed with the use of NIH Image software as described previously (Jung et al., 1998). Briefly, densitometric analyses were performed by encircling the cells with the freehand selection tool of the program. Images were inverted and the background was subtracted by using the automated function of the program. For calculation of relative density within perikarya and respective axonal neurites, these regions were recorded individually, and the densitometric value obtained for each was divided by the total value for both regions. For some analyses the axons were encircled with the freehand tool, and "plot profiles" were generated by using the program function. To determine the relative distribution of material along the periphery versus the center of perikarya, we encircled a representative location along the periphery 

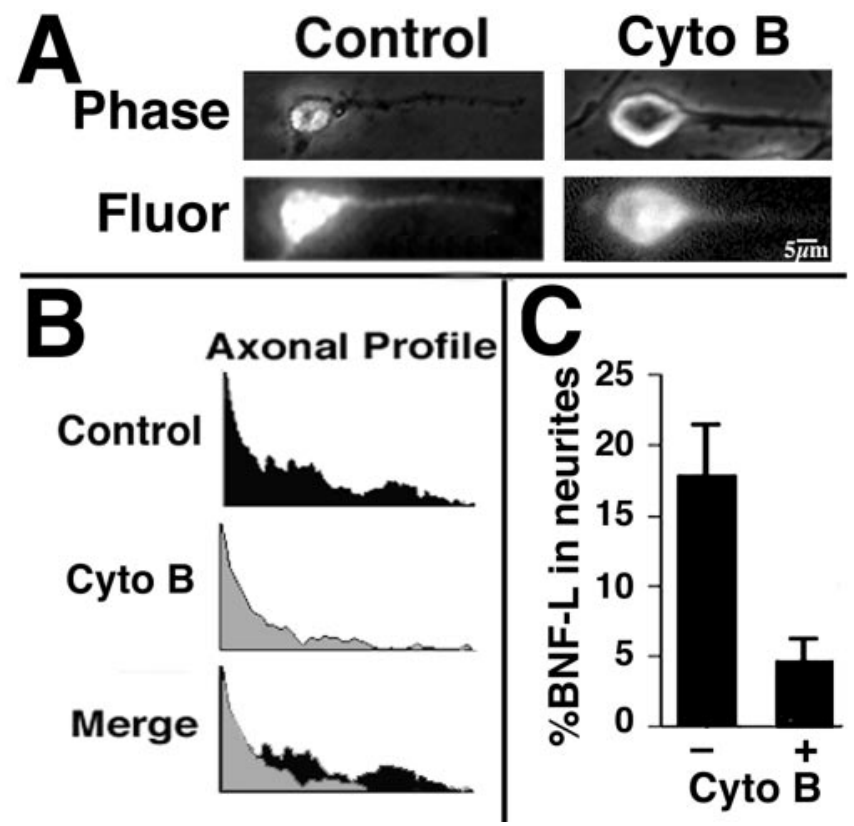

Figure 4. Cytochalsin B treatment inhibits transport of biotinylated NF subunits into axonal neurites. $A$, The panels present phase-contrast and corresponding epifluorescent images of representative cells microinjected with bNF-L or tracer alone and then fixed and immunostained for biotin at $1 \mathrm{hr}$ after injection; alternate cultures received $5 \mu \mathrm{m}$ cytochalasin B immediately after injection. $B$, We visualized the distribution of bNF-L (Axonal Profile) within cytochalasin B-treated and cytochalasin B-untreated axonal neurites for the presented images by encircling the axonal neurite from hillock to growth cone and invoking the plot profile function of NIH Image. Overlaying of plot profiles (Merge) from untreated and treated neurites highlights that both vinblastine and cytochalasin B inhibited translocation of bNF-L into neurites. C, The accompanying bar graph presents the percentage of bNF-L that had translocated into neurites within $1 \mathrm{hr}$ after microinjection (mean $\pm S D ; n \geq 3$ cells for each condition from $\geq 3$ separate experiments for a total of $\geq 9$ individual injected cells), calculated by dividing the fluorescent intensity within neurites by that within the neurite plus the corresponding cell body. Note that cytochalasin B markedly reduces the transport of biotinylated NF subunits into neurites.

by using the freehand selection tool and obtained the densitometric value. To yield an identically sized region from the center of the perikaryon, we then dragged the "selection box" from the periphery to a corresponding area within the center of the perikaryon without changing its size or shape and recorded the densitometric value. Values obtained from multiple cells were exported into Microsoft Excel (Redmond, WA) for calculation of ratios and statistical analyses. All ratios of relative bNF-L distribution were calculated individually for injected cells.

Overall translocation of GFP-tagged NF subunits expressed after transfection was quantified by dividing individual neurites into thirds and calculating the percentage of total axonal GFP within each third. We then determined the ratio of GFP within the distal third versus the central third in the presence and absence of pharmacological agents. We also monitored translocation of individual GFP-tagged particles by using two methods. Unlike microinjection in which the localization of bNF-L subunits can be visualized within minutes after injection, transfection under our conditions requires $18-24 \mathrm{hr}$ before sufficient GFP-tagged subunits have been expressed to allow reproducible visualization (Yabe et al., 1999, 2001a,b). Although GFP-tagged subunits typically have incorporated throughout the axonal NF network of many cells by this time, in those cells in which this has not yet occurred multiple GFP-tagged punctate and filamentous structures can be visualized readily along the periphery of axonal neurites. For real-time analyses of transport of these structures, digital images were captured at $5 \mathrm{~min}$ intervals before and after treatment with cytochalasin B, ML-7, or BDM. Sequential images were exported to QuickTime for video sequences (set at a speed of 3 frames/sec) or false-colored with either red or green. Merged images of sequential frames were created by overlaying false-colored images and rendering the overlaid image at 50\% opacity, using Adobe Photoshop
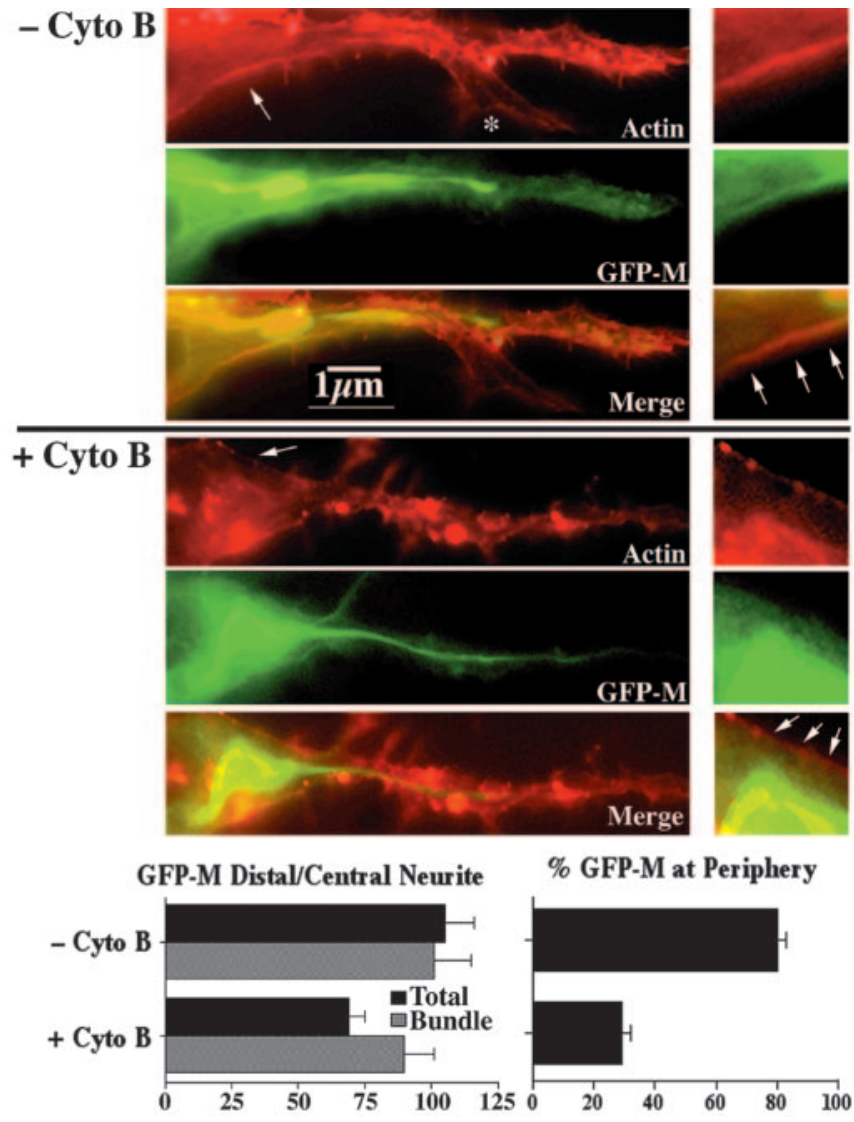

Figure 5. Cytochalasin B inhibits the distribution of GFP-tagged NF subunits. The panels present epifluorescent images of representative cells transfected with GFP-M and reacted with rhodamine-phalloidin before $(-C y$ to B) and after (+Cyto B) treatment with cytochalasin B. Note that cytochalasin $B$ reduced the total level of GFP-M within the distalmost region of the neurite but did not seem to reduce GFP-M levels within the central bundle. Also note the absence of GFP-tagged NFs from an actin-rich filopodium (asterisk), confirming discrete staining. The accompanying left graph presents densitometric analyses of the ratio of total and bundle-associated GFP-M in the distal third of axonal neurites versus the central third; these densitometric analyses confirm the visual impression that cytochalasin $B$ reduced overall GFP-M, but not bundled GFP-M, with distal axonal regions. The panels on the right present higher-magnification images of the region denoted by a small arrow in the image on the left. Arrows within the higher-magnification images indicate the peripheral region of the cell. The accompanying right graph presents densitometric analyses of the ratio of GFP-M at the periphery versus within central regions before and after cytochalasin B treatment. Note that cytochalasin $B$ reduced the localization of GFP-M along the periphery.

software; a reversal of whichever image was overlaid did not alter the merged image. Particles that had not translocated during a given interval therefore displayed a merged orange-yellow fluorescence, whereas those that had undergone translocation displayed either red or green fluorescence. The percentage of particles displaying either red or green fluorescence versus those displaying orange-yellow fluorescence was quantified at intervals before and after treatment. Translocation of GFP-tagged structures also was quantified by superimposing an arbitrary vertical line across the identical location in sequential images, which allowed us to determine the number of particles that had undergone either anterograde or retrograde translocation or had displayed no net translocation during a given interval; particles were considered to have undergone translocation provided they had shifted at least one full particle diameter between intervals (Yabe et al., 2001a).

\section{Results}

Translocation of NF subunits into axonal neurites in culture requires filamentous actin

Axonal neurites of NB2a/d1 neuroblastoma cells contain an array of NFs and filamentous actin (Fig. 1). Filamentous actin is con- 
centrated along the periphery and within microvilli of axonal neurites (Fig. 1). As shown previously (Yabe et al., 2001a,b), GFP-conjugated NF subunits incorporate into the endogenous NF array as demonstrated by the eventual colocalization of GFP with endogenous filamentous profiles, including the centrally situated NF bundle (Fig. 1). GFP-tagged short NFs and punctate structures also are observed throughout the axoplasm (Fig. 2). However, in neurites in which GFP-tagged NF subunits have not yet incorporated throughout the entire NF network, the majority (83.4 $\pm 6.2 \%)$ of GFP-tagged short NFs and punctate structures is localized toward the peripheral aspect of neurites, apparently along the inner surface of the axonal plasma membrane $(n=7$ transfected neurites; 27-60 individual GFP-tagged NFs and punctate structures were scored per neurite for a total of 256 structures). Sequential capturing of images has revealed that many such particles have undergone bidirectional translocation along the axonal neurite plasma membrane (Fig. 2) (a video sequence of particle translocation is presented as supplemental material, available at www.jneurosci.org).

Because translocation along the plasma membrane is consistent with the possibility that these GFP-tagged structures are undergoing transport via the actin submembrane cortex, we set out to determine whether or not the actin submembrane cortex mediated NF axonal transport. To test this hypothesis, we treated cells with 5 $\mu \mathrm{M}$ cytochalasin B for $1 \mathrm{hr}$, which disrupted the actin cortex (Fig. 3), and we monitored the influence of this treatment on translocation into axonal neurites of microinjected bNF-L and GFP-M expressed after transfection. Cytochalasin $\mathrm{B}$ reduced the level of biotinylated NF subunits or GFP-tagged subunits that translocated into axonal neurites (Figs. 4, 5). The most pronounced effect of cytochalasin $\mathrm{B}$ was on those NFs and NF-containing punctate structures that localized along the periphery of the perikaryon and neurite. Cytochalasin B selectively reduced the level of GFP fluorescence at the perikaryal periphery as compared with that within more central regions of the perikaryon. Cytochalasin B also reduced overall levels of GFP that had undergone transport into the distal region of neurites (Fig. 5). In addition, although cytochalasin B reduced overall transport of GFP-tagged NFs and NF-containing punctate structures into axons ( $p<0.045$; Student's $t$ test; $n=9$ untreated and 9 cytochalasin B-treated cells), it did not reduce significantly the level of GFP within the centrally situated NF bundle $(p<0.39$; Student's $t$ test; $n=5$ untreated and 5 treated cells) (Fig. 5). Comparison of the localization of GFP-tagged short NFs and punctate structures demonstrated that cytochalasin B rapidly inhibited their translocation within neurites (Fig. 6). These data
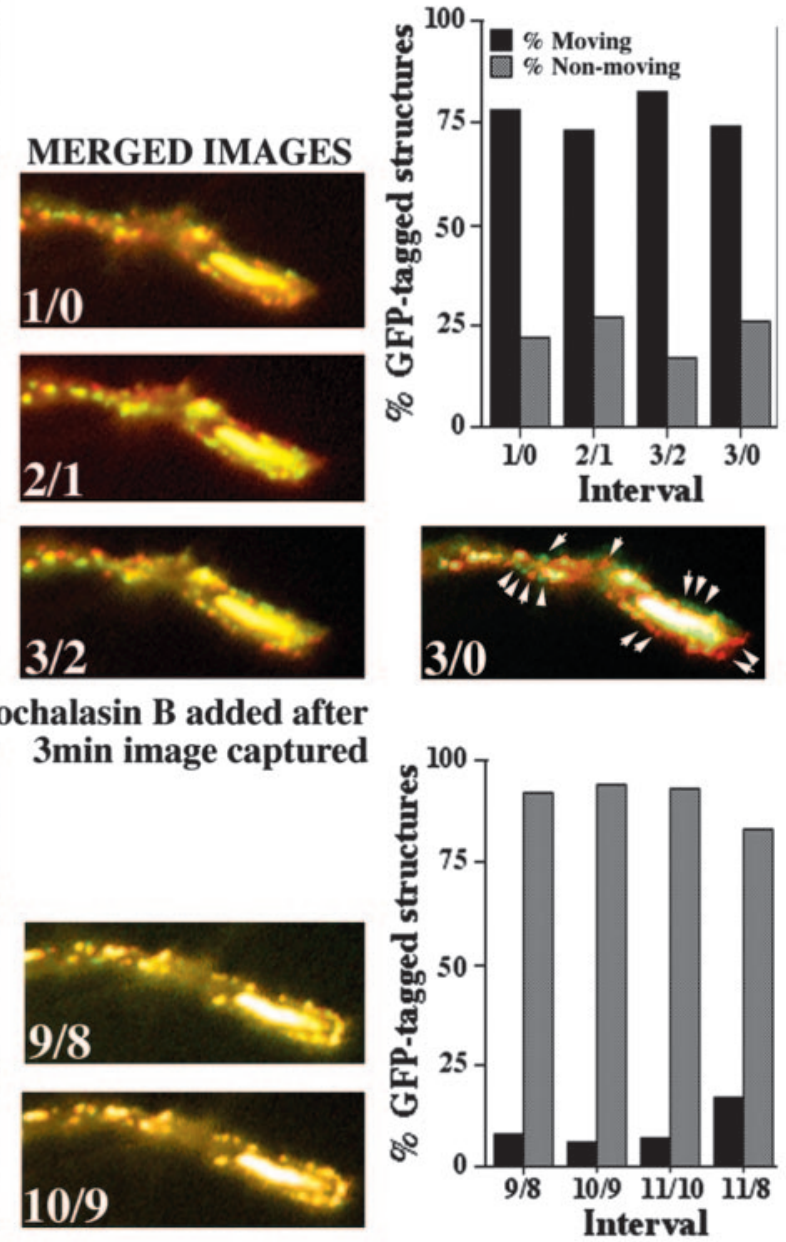

3min image captured
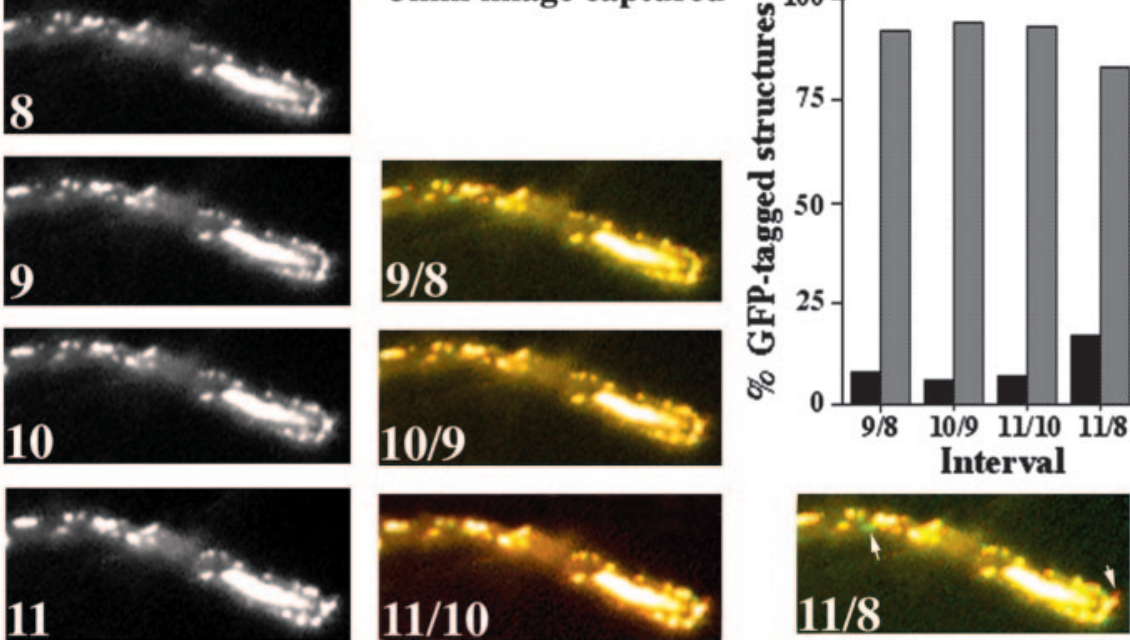

Figure 6. Cytochalasin B reduces translocation of GFP-tagged NFs and punctate structures. The panels present images captia images with either red or green and created a merged image for each interval; units in merged images correspond to the time A final merges before and after the addition of cytochalasin B denote representative GFP-tagged particles. The orange-yellow fluorescence for each interval before and after the addition of cytochalasin. Note that cytochalasin B markedly reduced the percentage of translocating particles.

suggest that the localization of short NFs and NF-containing punctate structures along the inner plasma membrane is attributable at least in part to their association with the actin cortex.

\section{Cytochalasin B perturbs NF transport in optic axons in situ}

To determine the physiological significance of these results obtained in neuroblastoma, we next monitored the influence of cytochalasin B on the rate and extent of NF axonal transport in situ. This was accomplished by intravitreal injection of 100 and $500 \mu \mathrm{M}$ cytochalasin $\mathrm{B}$ along with ${ }^{35} \mathrm{~S}$-methionine to radiolabel NF subunits metabolically, which then allows for monitoring of transport of newly synthesized NF subunits along optic axons. When this was performed, we observed that cytochalasin B inhib- 

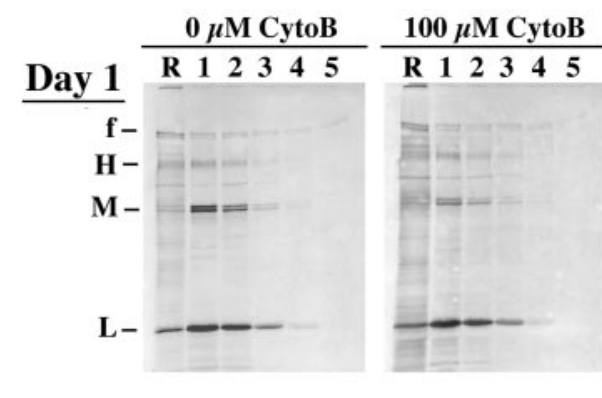

\section{\begin{tabular}{llllll}
500 & $\mu \mathrm{M}$ & $\mathrm{CytoB}$ \\
\hline R & 1 & 2 & 3 & 4 & 5
\end{tabular}}
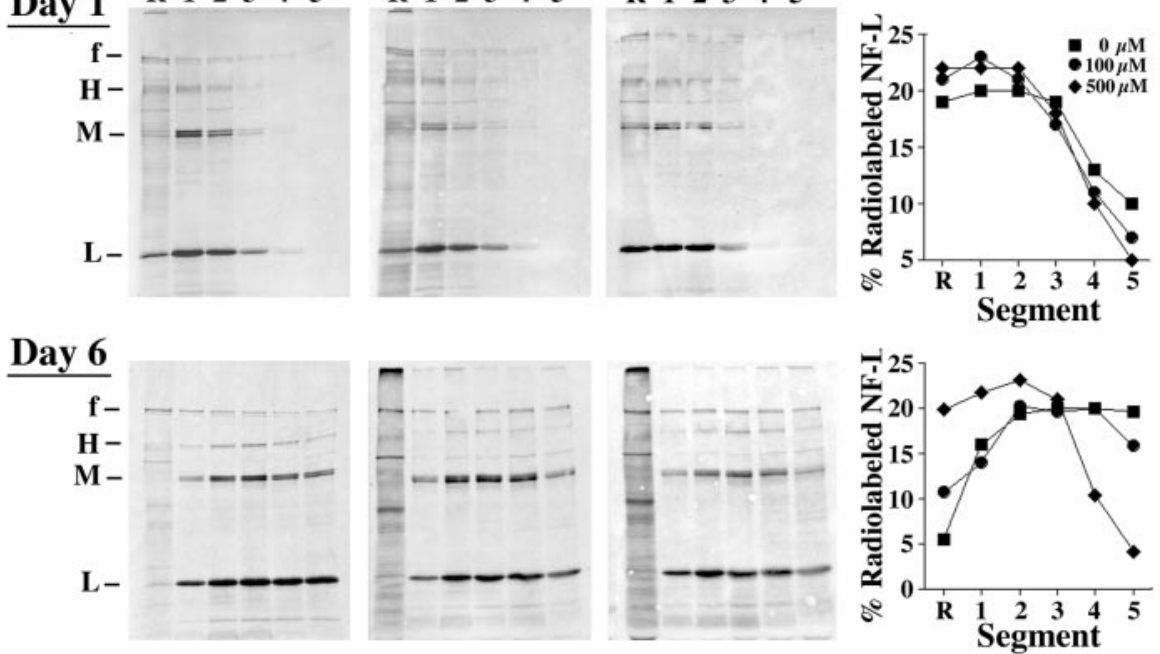

Figure 7. Cytochalasin B inhibits NF transport in optic axons. The panels present autoradiographs derived from SDS gels of material immunoprecipitated by R39 (which reacts with all NF subunits regardless of phosphorylation state) from Triton X-100insoluble fractions of retinas (R) and the first $5 \times 1.1 \mathrm{~mm}$ segments of optic axons harvested at 1 and $6 \mathrm{~d}$ after the injection of ${ }^{35} \mathrm{~S}$-methionine in the presence of 0,100 , or $500 \mu \mathrm{m}$ cytochalasin B as indicated. f, Front of the gel. The accompanying graphs present densitometric analyses of the distribution of radiolabeled NF-L, calculated for each segment as the percentage of total radiolabeled NF-L within all segments at each respective time interval. The percentage of distribution was calculated independently for each concentration of cytochalasin B. Note that cytochalasin B inhibited axonal transport of radiolabeled NFs.

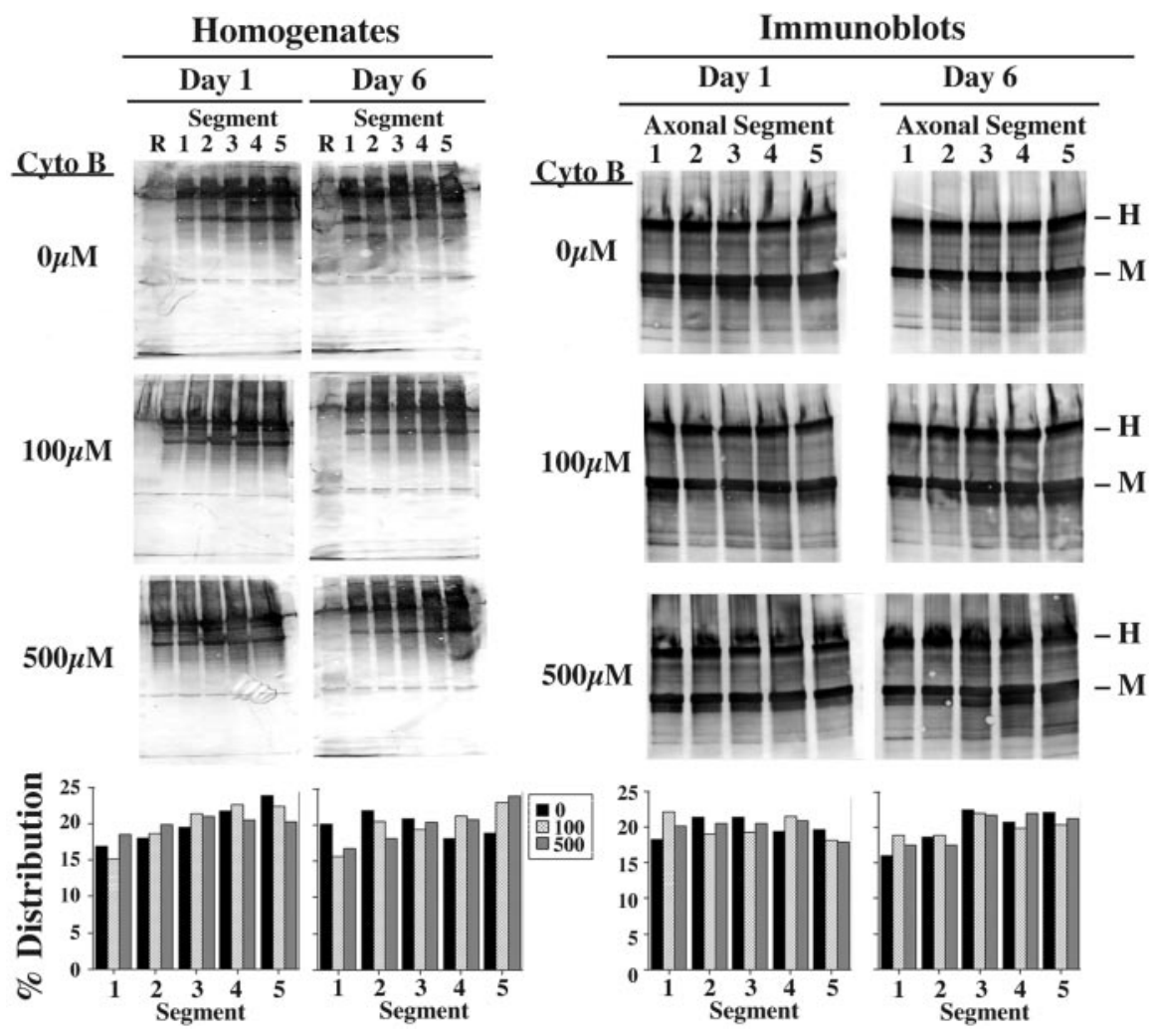

Figure 8. Cytochalasin B does not inhibit overall axonal transport or perturb the steady-state distribution of axonal NFs. The panels present autoradiographs or immunoblots, probed with $R 39$, of homogenates of retinas $(R)$ and the first $5 \times 1.1 \mathrm{~mm}$ segments of optic axons as described in the legend to Figure 7. The accompanying graphs present densitometric analyses of total radiolabeled proteins in autoradiographs or total NF-immunoreactive species in immunoblots. Note that cytochalasin treatment did not perturb overall axonal transport, nor did it reduce overall NF distribution under these conditions. ited NF axonal transport in a dose-response manner (Fig. 7). At day 1 after the injection of radiolabel in the absence of cytochalasin $\mathrm{B}$, the front of the wave of transporting NFs was recovered in segment 5 , whereas the peak was recovered in segment 2. In contrast, the front did not migrate so far and instead was recovered in segment 4 in mice coinjected with $\mathrm{cy}$ tochalasin $\mathrm{B}$. Considering the relative distance that NFs must have traveled for the front to be recovered within segments 5 and 4 , respectively, after $1 \mathrm{~d}$ of transport, the front of radiolabeled NFs had transported at the rate of $\sim 5 \mathrm{~mm} / \mathrm{d}$ in the absence of cytochalasin B but only $\sim 3.9$ $\mathrm{mm} / \mathrm{d}$ in mice receiving either concentration of cytochalasin B; cytochalasin B therefore reduced the axonal transport rate of the front by $\sim 20 \%$ over the first day after radiolabeling. It should be noted that, although the previously reported average rate of NF axonal transport (0.1-0.3 $\mathrm{mm} / \mathrm{d}$ ) is substantially slower that these rates, these faster transport rates are consistent with previous studies demonstrating that the front of the moving wave transports very rapidly (as fast as $14-100$ $\mathrm{mm} / \mathrm{d}$ ) over the first day (Lasek et al., 1993; Jung and Shea, 2004). In the absence of cytochalasin B the peak of radiolabeled NFs had reached segment 2 by day 1 after radiolabeling; although differences were apparent in the overall distribution of radiolabel in mice receiving either concentration of cytochalasin $\mathrm{B}$, the peak was recovered within segment 2 for these mice as well (Fig. 7). The inhibitory effect of cytochalasin B on NF axonal transport was more apparent by day 6 after radiolabeling. As anticipated (Lasek et al., 1993; Jung and Shea, 1999, 2004), the overall rate of NF axonal transport had declined considerably between days 1 and 6 in control mice not receiving cytochalasin $\mathrm{B}$. However, an even more pronounced decline in rate was observed in the presence of cytochalasin B. In mice receiving $500 \mu \mathrm{M} c y-$ tochalasin B, radiolabeled NFs had not undergone significant additional transport between days 6 and 1 ; at day 6 the front still was recovered within segment 4 and the peak still was recovered in segment 2 in optic pathways that had received $500 \mu \mathrm{M}$ cytochalasin B. In contrast, the front and peak were recovered within segments 5 and 3 in optic pathways receiving no cytochalasin B or $100 \mu \mathrm{M}$ cytochalasin B (Fig. 7). These inhibitory effects of cytochalasin B on NF transport were unlikely to be derived from overall toxicity and/or overall inhibition of axonal transport, because cytochalasin $\mathrm{B}$ under these conditions did 


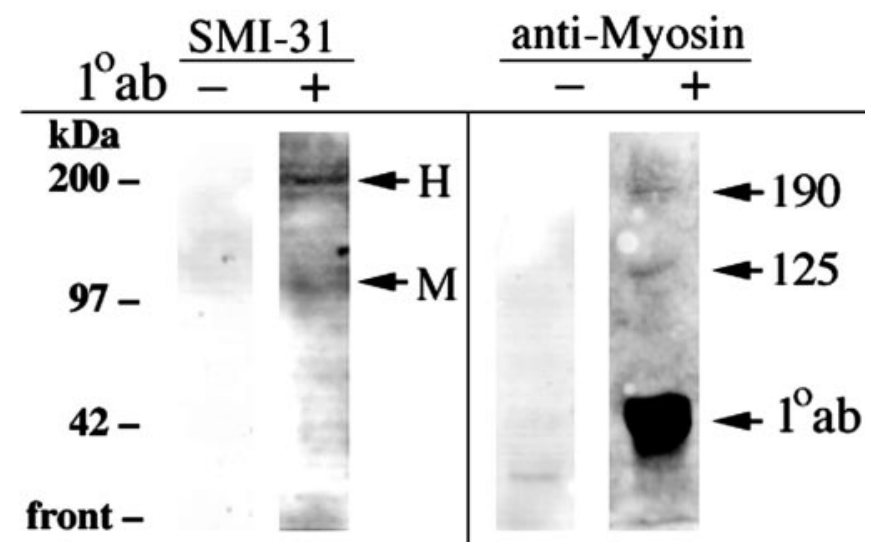

Figure 9. NF subunits coprecipitate with myosin. Homogenates of NB2a/d1 cells were subjected to immunoprecipitation either with anti-myosin (lanes designated by + ) or in the absence of any primary antibody (lanes designated by -). Immunoprecipitated material was subjected to SDS-gel electrophoresis, followed by immunoblot analysis with either SMI-31 or anti-myosin as indicated. Note the reactivity of immunoreactive species corresponding to the migratory positions of the intermediate chain of dynein and those of NF-M and NF-H (arrows) in blots reacted with SMI-31. In addition, note the presence of a $190 \mathrm{kDa}$ immunoreactive species (the expected migratory position of neuronal myosin) (Rao et al., 2002) in the blot reacted with anti-myosin; this blot also contains a prominent immunoreactive species at $\sim 125 \mathrm{kDa}$, which may represent a breakdown product of the $190 \mathrm{kDa}$ species or a novel myosin-immunoreactive species unique to these cells. As anticipated, the lgG derived from immunoprecipitation with anti-myosin also is stained prominently $\left(1^{\circ} \mathrm{ab}\right)$ by the goat secondary antibody used in antimyosin staining. Note too the lack of appreciable immunoreactivity with either SMI-31 or antimyosin for samples subjected to the immunoprecipitation procedure in the absence of primary antibody (-). The migratory position of molecular weight standards is indicated along the left.

not inhibit overall axonal transport of total radiolabeled proteins as revealed by autoradiographic analyses of homogenates of optic axon segments, nor did it perturb the distribution of total NFs along axons as revealed by immunoblot analyses of optic axon segments for total NF immunoreactivity (Fig. 8).

The actin-based motor, myosin, participates in anterograde NF transport

Perturbation of NF transport after treatment with cytochalasin B left open the possibility that the actin-based motor, myosin, may participate in NF subunit transport. This possibility was supported by the demonstration that NF-L is a ligand for myosin in axons (Rao et al., 2002). Consistent with the observed colocalization of myosin and NFs in vivo (Rao et al., 2002), myosin also was associated with NFs in differentiated NB2a/d1 cells. NF subunits were coprecipitated from NB2a/d1 cells with an anti-myosin antibody (Fig. 9). In addition, fluorescence microscopy demonstrated that myosin immunoreactivity colocalized with both the endogenous NF network as well as with GFP-tagged NF subunits expressed after transfection (Fig. 10). We took advantage of the ability to study transfected cells in real time to determine whether or not myosin regulated NF transport. To accomplish this, we treated transfected cells expressing GFP-M with the myosin light chain kinase inhibitor, ML-7, which previously has been used to assess the role of myosin in axonal transport (Morris and Hollenbeck, 1995; Bridgman, 1999), and with the myosin ATPase inhibitor BDM (Cramer and Mitchison, 1995). We then monitored the translocation of GFP-tagged NFs and punctate structures in sequentially captured images of axonal neurites of transfected cells. These analyses revealed that both ML-7 and BDM inhibited bidirectional axonal transport of GFPtagged structures within 30 min of treatment (Figs. 11, 12) (a video sequence of the inhibition of particle translocation by ML-7 is presented as supplemental material, available at www.jneurosci.org), suggesting that myosin participated in NF translocation within axonal neurites.

\section{Discussion}

The findings of the present study indicate that axonal transport and distribution of NFs are dependent at least in part on an intact actin submembrane network and that such transport, moreover, is facilitated by myosin. These findings support and extend the recent demonstration by Rao and colleagues (2002) that NFs are ligands for myosin and interact within axons in situ and that this interaction is essential for proper myosin transport and distribution of NFs within axons. Previous studies have shown that translocation of NFs into and along axons is dependent on an intact microtubule network (Jung et al., 1998; Yabe et al., 2000). Studies in culture (Yabe et al., 1999) and in situ (Prahlad et al., 2000; Yabe et al., 2000; Xia et al., 2003) additionally have demonstrated that the microtubule motor kinesin participates in anterograde NF transport, and cell-free analyses demonstrate that dynein can translocate NFs along microtubules (Shah et al., 2002). These findings collectively indicate that axonal transport of NFs is dependent on the integrity of the other major fibrous cytoskeletal constituents, actin filaments and microtubules, and their known motor proteins of myosin, kinesin, and dynein. Whether or not NF transport is entirely dependent on these motors or also uses one or more additional undisclosed motors (Xu and Tung, 2001) has not been determined. Notably, however, decades of analyses
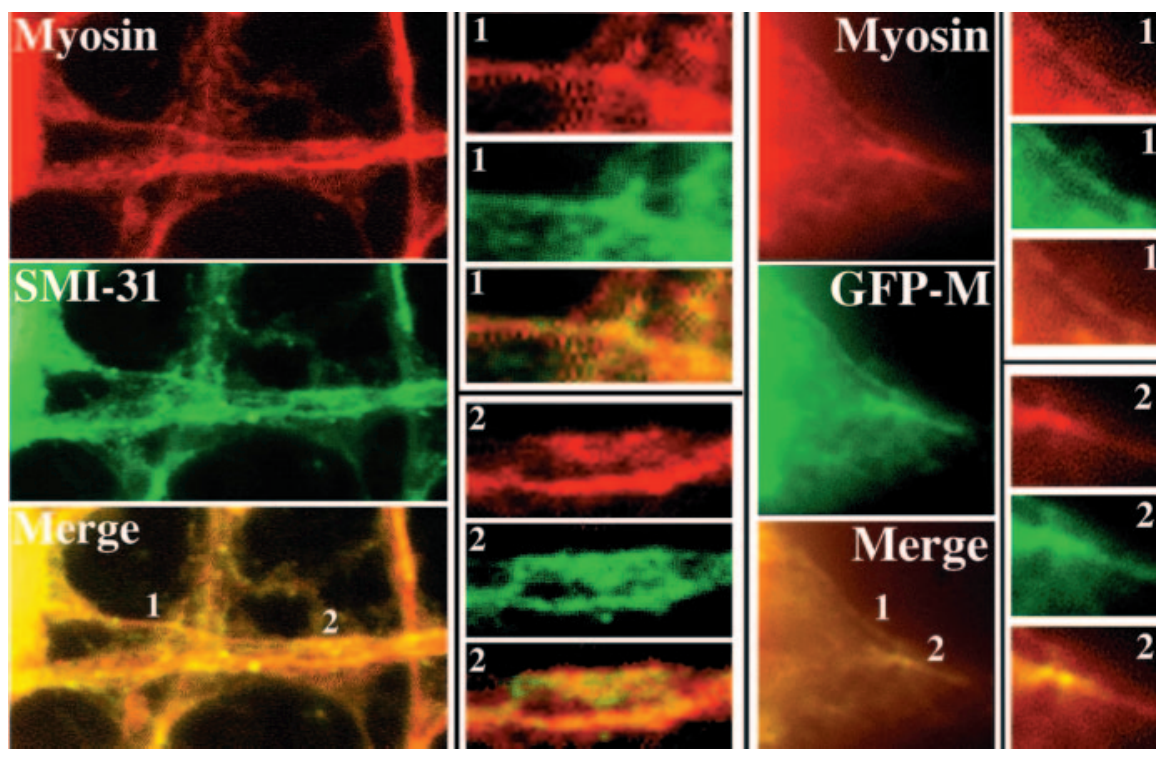

Figure 10. Myosin colocalizes with the endogenous NF network and with newly expressed (GFP-tagged) NFs. The panels present images of a cell double-immunostained with SMI-31 and anti-myosin and a cell transfected with GFP-M and immunostained with anti-myosin. Merged images were generated as described in Materials and Methods. Select regions (1,2) from each cell are presented at higher magnification. Note that myosin immunoreactivity colocalizes with both SMI-31 immunoreactivity and GFP-M. 

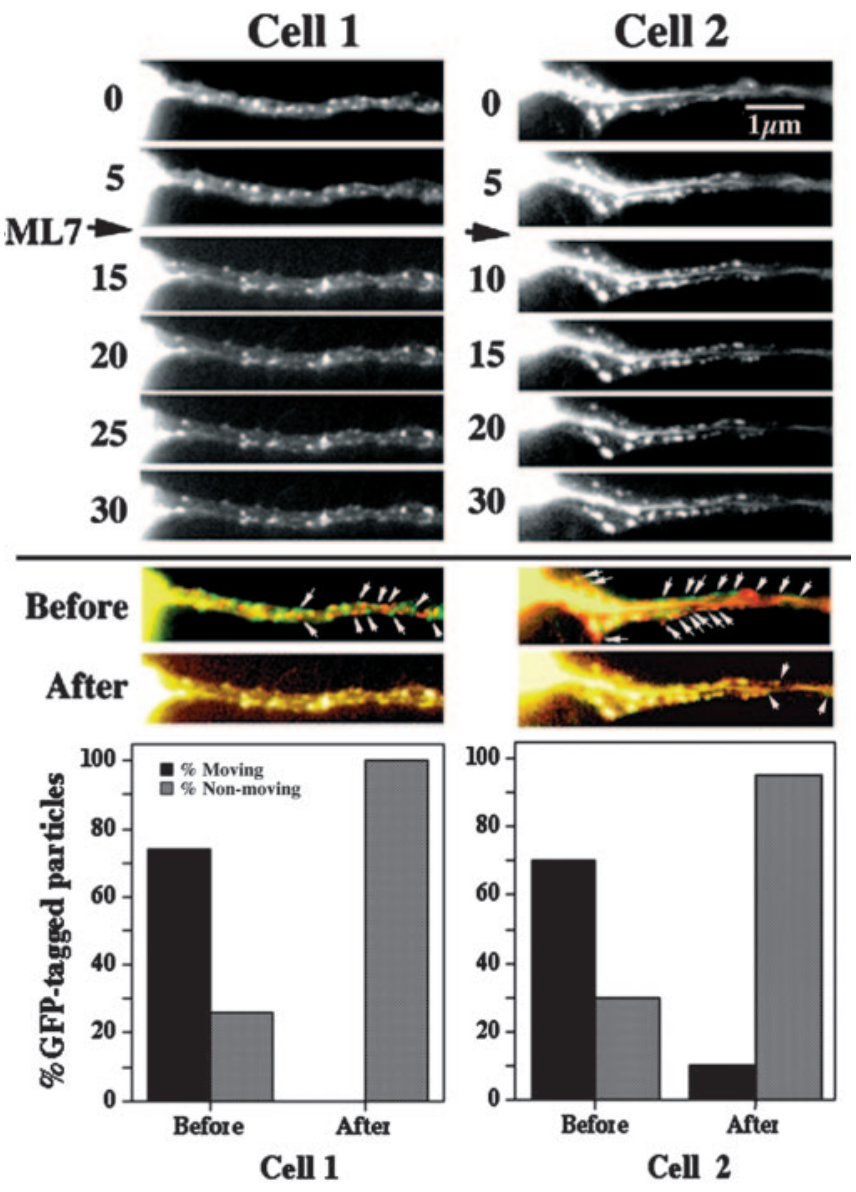

Figure 11. ML-7 inhibits bidirectional transport of GFP-tagged NFs and punctate structures. Sequential images captured at the indicated intervals of axonal neurites of two transfected cells before and after the addition (arrow) of $300 \mathrm{~nm} \mathrm{ML-7}$ are shown. Small arrows denote representative GFP-tagged structures. As described in Materials and Methods, two sequential images before the addition of ML-7 were false-colored with either red and green, and a merged image was created (Before); the same procedure was performed for two sequential images after the addition of cytochalasin B (After). Particles that had not translocated during the interval between the capture of images displayed a merged orange-yellow fluorescence, whereas those that had undergone translocation displayed either red or green fluorescence. The accompanying graphs present the percentage of particles displaying either red or green fluorescence versus those displaying orange-yellow fluorescence. Note that ML-7 reduced the percentage of translocating particles. A video sequence of the effect of ML-7 on GFP-tagged particle translocation is presented as supplemental material (available at www.jneurosci.org). The depicted cells correspond to those presented in Figure 2 before the addition of ML-7.

have not elucidated any motors specifically mediating so-called slow axonal transport, and the present findings, taken together with previous studies, demonstrate that NFs apparently undergo translocation by each of the major classes of known motors (kinesin, dynein, and myosin). Because each of these motors mediates fast axonal transport of other constituents (for review, see Gallant, 2000), these findings support the hypothesis of Ochs (1975) that a single motor system could mediate multiple transport rates by varying the length of time that cargo remains associated with its motor(s) (for review, see Shea and Yabe, 2000; Shea and Flanagan, 2001). Consistent with this view, real-time analyses of NF subunit transport demonstrate that NF subunits can undergo periodic bursts of fast transport interspersed by prolonged periods of nonmotility, which averages out to an overall transport rate consistent with slow axonal transport (Prahlad et al., 2000; Roy et al., 2000; Wang et al., 2000; Ackerley et al., 2003)

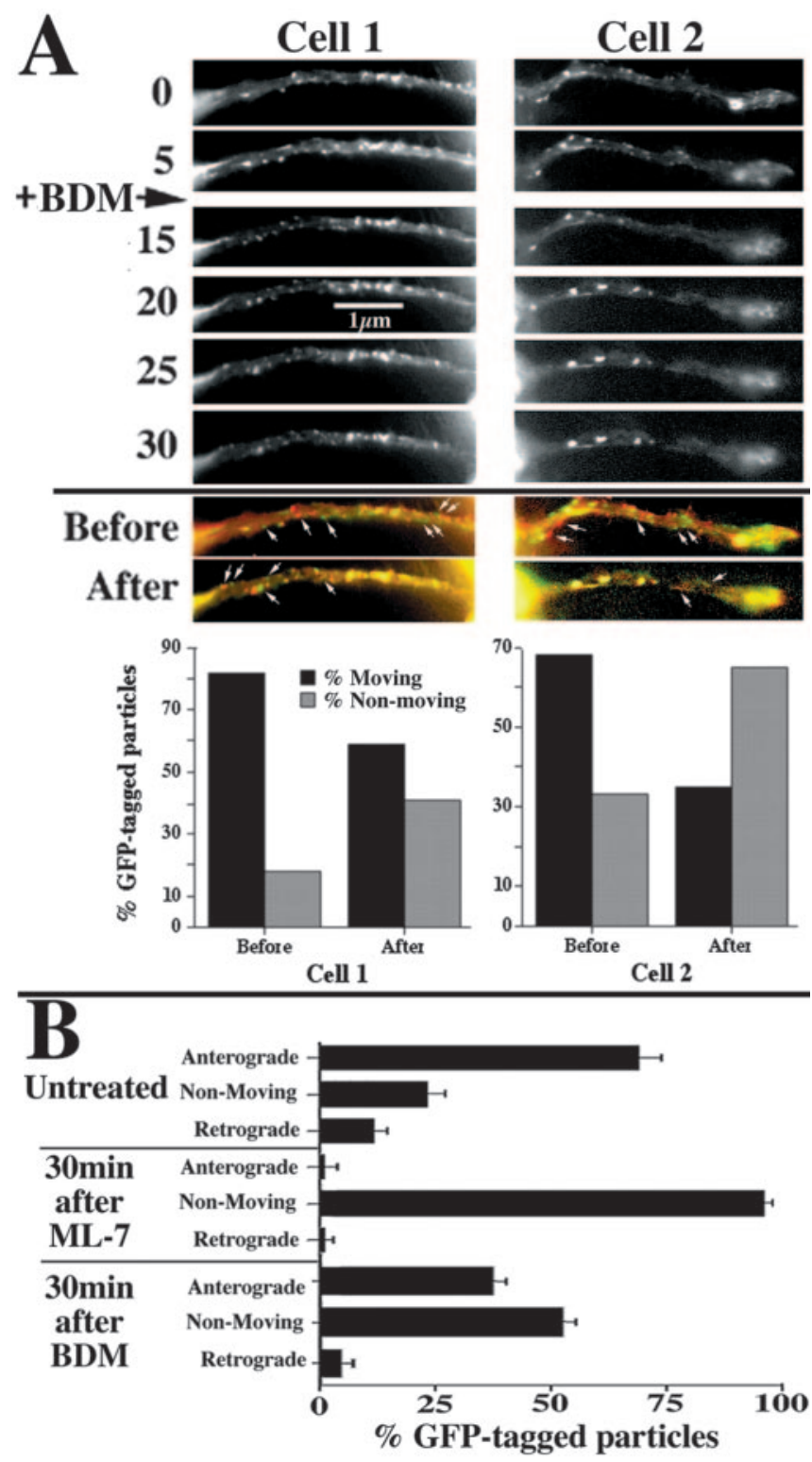

Figure 12. BDM and ML-7 decrease bidirectional transport of GFP-tagged particles. A, Sequential images from two representative transfected cells captured at the indicated intervals before and after the addition (arrow) of $20 \mathrm{~mm}$ BDM. Small arrows denote representative GFPtagged structures. The accompanying graphs present the percentage of particles displaying either red or green fluorescence versus those displaying orange-yellow fluorescence. Note that ML-7 reduced the percentage of translocating particles. $B$, The graph depicts the percentage (mean \pm SEM; $n \geq 3$ neurites under each condition from multiple experiments) of GFP-tagged structures undergoing anterograde transport, retrograde transport, or exhibiting no motion either before or 25-30 min after the addition of ML-7 or BDM as described in A. Note that both $\mathrm{ML}-7$ and BDM reduce the bidirectional transport of GFP-tagged structures.

(for review, see Brady, 2000; Chou and Goldman, 2000; Shah and Cleveland, 2002; Helfand et al., 2003a,b).

Although NFs undergo predominantly anterograde axonal transport (for review, see Shea and Yabe, 2000), many studies in culture and in situ also demonstrate that some NFs undergo retrograde subunit transport (Glass and Griffin, 1991; Watson et al., 1993; Koehnle and Brown, 1999; Yabe et al., 1999, 2001a; Roy et al., 2000; Shah et al., 2000; Wang et al., 2000). Retrograde NF transport may be mediated by the retrograde motor dynein (Shah et al., 2000). In addition to mediating retrograde transport of 
axonal constituents, however, dynein also translocates microtubules with their plus ends leading by interacting via its cargo domain with the actin cortex; because dynein cannot translocate the actin cortex, it instead translocates its associated microtubule in an anterograde direction into and along axons (Dillman et al., 1996a,b; Ahmad et al., 1998). This putative mechanism is supported by localization of dynein and dynactin along the cell cortex (Vallee and Sheetz, 1996; Hirokawa, 1998). Any NF subunits associated with such moving microtubules therefore also would be propelled in an anterograde direction by dynein-mediated microtubule transport (Susalka and Pfister, 2000). Association of NFs with microtubules, including such moving microtubules, could be mediated either by direct association (Miyasaka et al., 1993; Shah et al., 2000) or linkage via additional microtubule motor proteins (Liao and Gundersen, 1998; Shah et al., 2000), other microtubule-associated proteins (Miyata et al., 1986; Hirokawa et al., 1988), and/or NF-associated proteins such as plectins (Nixon, 1998; Yang et al., 1999; Herrmann and Aebi, 2000). Notably, this line of reasoning encompasses the concept that kinesin may serve to link NF subunits with microtubules (Liao and Gundersen, 1998) in addition to (or instead of) actively translocating microtubules (Yabe et al., 1999, 2000).

Although not investigated directly herein, polarity of the actin cortex (Morris and Hollenbeck, 1995) may be an important factor in maintaining net anterograde NF subunit transport as well as net delivery of microtubules into axons by dynein (Ahmad et al., 1998). Notably, coprecipitation of NF subunits by antimyosin and colocalization of NFs with myosin with axons [Rao et al. (2002); this work] suggest that some NFs may interact directly with, and undergo translocation along the actin cortex via, myosin without the need for dynein-mediated microtubule transport. Myosin Va also binds directly to kinesin (Huang et al., 1999), leaving open the possibility that myosin may link NFs to kinesin as well as to actin (for review, see Bridgman, 2004; Brown and Bridgman, 2004). In addition, myosin can transport microtubules (Waterman-Storer and Salmon, 1997); NFs therefore may undergo transport as cargo of microtubules that are driven by myosin as well as by dynein. It remains possible that kinesin, dynein, and myosin each participate in NF transport (Helfand et al., 2003a,b), perhaps with regional specificity and/or selectivity for various NF phospho-isoforms.

These findings, taken together with previous studies, afford some speculation regarding the role of actin-based translocation of NF subunits into and along axonal neurites. A delay of 18-24 $\mathrm{hr}$ is required for reproducible quantification of the distribution of GFP-tagged NF subunits (Yabe et al., 1999, 2001a,b). This complicates determination of the distribution of newly expressed NF subunits, because newly expressed subunits undergo translocation into axonal neurites within as little as $15 \mathrm{~min}$ (Jung et al., 1998). Accordingly, GFP-tagged NF subunits eventually incorporate throughout the entire endogenous NF array (Yabe et al., $2001 a, b)$. Consequently, in the majority of transfected cells, GFPtagged NF subunits are dispersed relatively throughout the entire perikarya and neurites of most cells by the time they can be visualized. However, in those neurites in which GFP-tagged subunits had not yet dispersed throughout the entire neurite, the majority of motile GFP-tagged NFs and punctate structures were localized along the inner plasma membrane surface. Because GFP-tagged particles provide an index of newly expressed subunits, it suggests that rapid initial delivery of NFs/NF-containing punctate structures may be via interactions with the actin submembrane skeleton. Moreover, perturbation of the actin network with cytochalasin B disrupted translocation of those GFP-tagged structures along the membrane but did not perturb those within the central NF bundle. These findings suggest that small NFs and punctate structures may undergo translocation via interactions with the actin cortex, whereas bundled NFs may not.

Previous studies demonstrate that bundled NFs undergo transport/turnover more slowly than do more peripherally situated NFs/punctate structures and that NF subunits within bundles display increased C-terminal phosphorylation versus more peripherally situated subunits (Yabe et al., 2001a,b). It also has been suggested that bundled NFs represent those NFs that have dissociated from their motor system(s) and have undergone NF-NF interactions that compete with transport (Shea and Yabe, 2000). One interpretation of these findings is that relatively nonphosphorylated NFs/punctate structures may undergo translocation into and along axons via interactions with the actin cortex. Consistent with this possibility is that cytochalasin B treatment perturbed transport of the front of the radiolabeled wave of NF subunits (which is enriched in relatively less-phosphorylated NFs) in optic axons in situ substantially more than it perturbed transport of the peak of the radiolabeled wave (which is enriched in extensively phosphorylated NFs) (Jung et al., 2000a,b). The preferential interaction of less-phosphorylated NF subunits with kinesin (Yabe et al., 1999, 2000; Chan et al., 2004) also remains consistent with this possibility, because kinesin may link hypophosphorylated NFs to moving microtubules that themselves translocate along actin via dynein-mediated interactions. We have hypothesized previously that bundled NFs may have to dissociate from the centrally situated bundle and/or undergo key dephosphorylation events to interact with microtubule-based motors and continue axonal transport (Shea and Yabe, 2000); the findings of the present study suggest that such events also may be required for interaction with, and transport via, the actin cortex. Of interest would be to determine the relative affinity of kinesin, dynein, and myosin for various NF phospho-isoforms.

The findings of the present study attribute additional importance of the actin/myosin system in development of the nervous system. Although this system is known to mediate the initial outgrowth of axons (Lefcort and Bentley, 1989; Shea, 1990; Wang and Jay, 1996; Bridgman and Elkin, 2000; Dent and Gertler, 2003; Bridgman, 2004; Brown and Bridgman, 2004), the demonstration that axonal transport of NFs is dependent on the integrity of the actin cytoskeleton and on the function of its motor myosin indicates that this system also provides the essential framework for NF-mediated stabilization of developing axons.

\section{References}

Ackerley S, Thornhill P, Grierson AJ, Brownlees J, Anderton BH, Leigh PN, Shaw CE, Miller CC (2003) Neurofilament heavy chain side-arm phosphorylation regulates axonal transport of neurofilaments. J Cell Biol 161:489-495.

Ahmad FJ, Echeverri RB, Vallee RB, Baas PW (1998) Cytoplasmic dynein and dynactin are required for the transport of microtubules into the axon. J Cell Biol 140:391-401.

Baas PW, Deitch JS, Black MM, Banker GA (1988) Polarity orientation of microtubules in hippocampal neurons: uniformity in the axon and nonuniformity in the dendrite. Proc Natl Acad Sci USA 85:8335-8339.

Brady ST (2000) Neurofilaments run sprints not marathons. Nat Cell Biol 2:E43-E45.

Bridgman PC (1999) Myosin Va movements in normal and dilute-lethal axons provide support for a dual filament motor complex. J Cell Biol 146:1045-1060.

Bridgman PC (2004) Myosin-dependent transport in neurons. J Neurobiol 58:164-174.

Bridgman PC, Elkin LL (2000) Axonal myosins. J Neurocytol 29:831-841.

Brown ME, Bridgman PC (2004) Myosin function in nervous and sensory systems. J Neurobiol 58:118-130. 
Chan WK, Dickerson A, Otriz D, Pimenta A, Moran C, Malik K, Motil J, Snyder S, Pant HC, Shea TB (2004) Mitogen-activated protein kinase regulates neurofilament axonal transport. J Cell Sci, in press.

Chou YH, Goldman RD (2000) Intermediate filaments on the move. J Cell Biol 150:F101-F106.

Cramer LP, Mitchison TJ (1995) Myosin is involved in the post-mitotic cell spreading. J Cell Biol 131:179-189.

Dent EW, Gertler FB (2003) Cytoskeletal dynamics and transport in growth cone motility and axon guidance. Neuron 40:209-227.

Dillman JF, Dabney LP, Pfister KK (1996a) Cytoplasmic dynein is associated with slow axonal transport. Proc Natl Acad Sci USA 93:141-144.

Dillman JF, Dabney LP, Karki S, Paschal BM, Holzbaur EL, Pfister KK (1996b) Functional analysis of dynactin and cytoplasmic dynein in slow axonal transport. J Neurosci 16:6742-6752.

Frappier T, Regnouf F, Pradel L-A (1987) Binding of brain spectrin to the 70 kDa neurofilament subunit protein. Eur J Biochem 169:651-657.

Frappier T, Stetzkowski-Marden F, Pradel L-A (1991) Interaction domains of neurofilament light chain and brain spectrin. Biochem J 275:521-527.

Galbraith JA, Gallant PE (2000) Axonal transport of tubulin and actin. J Neurocytol 29:889-911.

Gallant PE (2000) Axonal protein synthesis and transport. J Neurocytol 29:779-782.

Glass JD, Griffin JW (1991) Neurofilament redistribution in transected nerves: evidence for bidirectional transport of neurofilaments. J Neurosci 11:3146-3154.

Gross SP, Welte MA, Block SM, Wieschaus EF (2000) Dynein-mediated cargo transport in vivo: a switch controls travel distance. J Cell Biol 148:945-955.

Heidemann SR, Landers JM, Hamborg MA (1981) Polarity orientation of axonal microtubules. J Cell Biol 91:661-665.

Helfand BT, Chang L, Goldman RD (2003a) The dynamic and motile properties of intermediate filaments. Annu Rev Cell Dev Biol 19:445-467.

Helfand BT, Loomis P, Yoon M, Goldman RD (2003b) Rapid transport of neural intermediate filament protein. J Cell Sci 116:2345-2359.

Herrmann H, Aebi U (2000) Intermediate filaments and their associates: multitalented structural elements specifying cytoarchitecture and cytodynamics. Curr Opin Cell Biol 12:79-90.

Hirokawa N (1993) Axonal transport and the cytoskeleton. Curr Opin Neurobiol 3:724-731.

Hirokawa N (1998) Kinesin and dynein superfamily proteins and the mechanism of organelle transport. Science 279:519-526.

Hirokawa N, Hisanaga S, Shiomura Y (1988) MAP2 is a component of crossbridges between microtubules and neurofilaments in the neuronal cytoskeleton: quick-freeze, deep-etch immunoelectron microscopy, and reconstitution studies. J Neurosci 8:2769-2779.

Huang JD, Brady ST, Richards BW, Stenoien D, Resau JH, Copeland NG, Jenkins NA (1999) Direct interaction of microtubule- and actin-based transport motors. Nature 397:267-270.

Jung C, Shea TB (1999) Regulation of neurofilament axonal transport by phosphorylation in optic axons in situ. Cell Motil Cytoskeleton 42:230-240.

Jung C, Yabe J, Wang F-S, Shea TB (1998) Neurofilaments are present within axonal neurites before incorporation into Triton-insoluble structures. Cell Motil Cytoskeleton 40:44-58.

Jung C, Yabe JT, Shea TB (2000a) C-terminal phosphorylation of the high molecular weight neurofilament subunit correlates with decreased neurofilament axonal transport velocity. Brain Res 856:12-19.

Jung C, Yabe JT, Lee S, Shea TB (2000b) Hypophosphorylated neurofilament subunits undergo axonal transport faster than extensively phosphorylated subunits. Cell Motil Cytoskeleton 47:120-129.

Jung CJ, Shea TB (2004) Neurofilament subunits undergo more rapid translocation within retinas than in optic axons. Mol Brain Res 122:188192.

Koehnle TJ, Brown A (1999) Slow axonal transport of neurofilament protein in cultured neurons. J Cell Biol 144:447-458.

Kuznetsov SA, Langford GM, Weiss DG (1992) Actin-dependant organelle movement in squid axoplasm. Nature 356:722-725.

Lasek RJ, Paggi P, Katz MJ (1993) The maximum rate of neurofilament transport in axons: a view of molecular transport mechanisms continuously engaged. Brain Res 616:58-64.

Lefcort F, Bentley D (1989) Organization of cytoskeletal elements and or- ganelles preceding growth cone emergence from an identified neuron in situ. J Cell Biol 108:1737-1749.

Liao G, Gundersen GG (1998) Kinesin is a candidate for cross-bridging microtubules and intermediate filaments. J Biol Chem 273:9797-9803.

Martin MA, Hurd DD, Saxton WM (1999) Kinesins in the nervous system. Cell Mol Life Sci 56:200-216.

Miyasaka H, Okabe S, Ishiguro K, Uchida T, Hirokawa N (1993) Interaction of the tail domain of high molecular weight subunits of neurofilaments with the $\mathrm{COOH}$-terminal region of tubulin and its regulation by tau protein kinase II. J Biol Chem 268:22695-22702.

Miyata Y, Hoshi M, Nishida E, Minami Y, Sakai H (1986) Binding of microtubule-associated protein 2 and tau to the intermediate filament reassembled from neurofilament $70 \mathrm{kDa}$ subunit protein. J Biol Chem 261:13026-13030.

Morris RL, Hollenbeck PJ (1995) Axonal transport of mitochondria along microtubules and F-actin in living vertebrate neurons. J Cell Biol 131:1315-1326.

Nixon RA (1992) Slow axonal transport. Curr Opin Cell Biol 4:8-14.

Nixon RA (1998) Dynamic behavior and organization of cytoskeletal proteins in neurons: reconciling old and new findings. BioEssays 20:798-807.

Ochs S (1975) A unitary concept of the axoplasmic transport based on the transport filament hypothesis. In: Recent advances in myology (Bradley WG, ed), pp 189-194. Amsterdam: Excerpta Medica.

Prahlad V, Helfand BT, Langford GM, Vale RD, Goldman RD (2000) Fast transport of neurofilament protein along microtubules. J Cell Sci 113:3939-3946.

Rao MV, Engle LJ, Mohan PS, Yuan A, Qiu D, Cataldo AM, Hassinger L, Jacobsen S, Lee VM-Y, Andreadis A, Julien J-P, Bridgman PC, Nixon RA (2002) Myosin Va binding to neurofilaments is essential for correct myosin Va distribution and transport and neurofilament density. J Cell Biol 159:279-289.

Roy S, Coffee P, Smith G, Liem RKH, Brady ST, Black MM (2000) Neurofilaments are transported rapidly but intermittently in axons: implications for slow axonal transport. J Neurosci 20:6849-6861.

Shah JV, Cleveland DW (2002) Slow axonal transport: fast motors in the slow lane. Curr Opin Cell Biol 14:58-62.

Shah JV, Flanagan LA, Janmey PA, Leterrier JF (2000) Bidirectional translocation of neurofilaments along microtubules is mediated in part by dynein/dynactin. Mol Biol Cell 11:3495-3508.

Shea TB (1990) Neuritogenesis in mouse NB2a/d1 neuroblastoma cells: triggering by calcium influx and involvement of actin and tubulin dynamics. Cell Biol Int Rep 14:967-979.

Shea TB (2000) Microtubule motors, phosphorylation, and axonal transport of neurofilaments. J Neurocytol 29:873-887.

Shea TB, Beermann ML (1994) Sequential requirements for neurofilaments, microtubules, and the microtubule-associated proteins MAP1B and tau in axonal neurite initiation, elongation, and stabilization. Mol Cell Biol 5:863-875.

Shea TB, Flanagan L (2001) Kinesin, dynein, and neurofilament transport. Trends Neurosci 24:644-648.

Shea TB, Yabe J (2000) Occam's razor slices through the mysteries of neurofilament axonal transport: can it really be so simple? Traffic 1:522-523.

Shea TB, Sihag RK, Nixon RA (1988) Neurofilament triplet proteins of NB2a/d1 neuroblastoma: posttranslational modification and incorporation into the cytoskeleton during differentiation. Dev Brain Res 50:142-146.

Shea TB, Sihag RK, Nixon RA (1990) Dynamics of phosphorylation and assembly of the high molecular weight neurofilament protein subunit in NB2a/d1 neuroblastoma. J Neurochem 55:1784-1792.

Shea TB, Dahl DC, Nixon RA, Fischer I (1997) Triton-soluble phosphovariants of the heavy neurofilament subunit in developing and mature mouse central nervous system. J Neurosci Res 48:515-523.

Susalka SJ, Pfister KK (2000) Cytoplasmic dynein subunit heterogeneity: implications for axonal transport. J Neurocytol 29:819-829.

Vale R, Fletterick R (1997) The design plan of kinesin motors. Annu Rev Cell Dev Biol 13:745-777.

Vallee RB, Sheetz MP (1996) Targeting of motor proteins. Science 271:1539-1544.

Wang FS, Jay DG (1996) Chromophore-assisted laser inactivation (CALI): probing protein function in situ with a high degree of spatial and temporal resolution. Trends Cell Biol 6:442-445. 
Wang L, Ho CL, Sun DM, Liem RKH, Brown A (2000) Rapid movement of axonal neurofilaments interrupted by prolonged pauses. Nat Cell Biol 2:137-141.

Waterman-Storer CM, Salmon ED (1997) Actomyosin-based retrograde flow of microtubules in the lamella of migrating epithelial cells influences microtubule dynamic instability and turnover and is associated with microtubule breakage and treadmilling. J Cell Biol 139:417-434.

Watson DF, Glass JD, Griffin JW (1993) Redistribution of cytoskeletal proteins in mammalian axons disconnected from their cell bodies. J Neurosci 13:4354-4360.

Xia CH, Roberts EA, Her LS, Liu X, Williams DS, Cleveland DW, Goldstein LS (2003) Abnormal neurofilament transport caused by targeted disruption of neuronal kinesin heavy chain KIF5A. J Cell Biol 161:55-66.

$\mathrm{Xu} Z$, Tung VW (2001) Temporal and spatial variations in slow axonal transport velocity along peripheral motoneuron axons. Neuroscience 102:193-200.
Yabe JT, Pimenta A, Shea TB (1999) Kinesin-mediated transport of NF protein oligomers in growing axons. J Cell Sci 112:3799-3814.

Yabe JT, Jung C, Chan WK, Shea TB (2000) Phospho-dependent association of neurofilament proteins with kinesin in situ. Cell Motil Cytoskeleton 45:249-262.

Yabe JT, Chan WK, Chylinski TM, Lee S, Pimenta AF, Shea TB (2001a) The predominant form in which neurofilaments subunits undergo axonal transport varies during axonal initiation, elongation, and maturation. Cell Motil Cytoskeleton 48:61-83.

Yabe JT, Chylinski TM, Wang F-S, Pimenta AF, Kattar SD, Linsley M-D Chan WK, Shea TB (2001b) Neurofilaments consist of distinct populations that can be distinguished by C-terminal phosphorylation, bundling, and axonal transport rate in growing axonal neurites. J Neurosci 21:2195-2205.

Yang Y, Bauer C, Strasser G, Wollman R, Julien JP, Fuchs E (1999) Integrators of the cytoskeleton that stabilize microtubules. Cell 98:229-238. 